\title{
Vertical Electron Transport in GaN/AlGaN Heterostructures
}

\begin{abstract}
A. ReKLaitis*
Semiconductor Physics Institute, A. Goštauto 11, 01108 Vilnius, Lithuania

Nonequilibrium dc and large-signal ac vertical electron transport in GaN/AlGaN heterostructures is investigated by Monte Carlo simulations. The symmetric two-barrier GaN/AlGaN heterostructures are studied. The results of simulations show that polarization charges have a profound effect on dc and large-signal ac characteristics of vertical electron transport in GaN/AlGaN heterostructures. Under certain composition, geometry and doping profile, the $\mathrm{GaN} / \mathrm{AlGaN}$ heterostructures may become bipolar, i.e., the inversion layers may originate at heterointerfaces due to strong built-in electric fields, which are induced by polarization charges.
\end{abstract}

PACS numbers: 73.40.Kp, 72.20.-i, 72.30.+q

\section{Introduction}

The wide band-gap semiconductors have attracted a great interest for possible application in future high power, high frequency electronics. A detailed understanding of electron transport processes underlying the electrical characteristics of $\mathrm{GaN} / \mathrm{AlGaN}$ heterostructures is an essential requirement for the successful design of the devices. The features of vertical transport in GaN/AlGaN heterostructures is of fundamental importance for the design of a large number of electronic and photonic devices. An essential circumstance, which has a significant influence on the electrical characteristics of GaN/AlGaN, is the strong polarization charges at $\mathrm{GaN} / \mathrm{AlGaN}$ heterointerfaces. In the present work, vertical electron transport in the GaN/AlGaN heterostructures is studied by the Monte Carlo simulations. Direct current and large-signal ac electron transport in symmetric double-barrier heterostructures is investigated. The results of simulations predict that GaN/AlGaN material system is well suited for the design of heterostructure-barrier varactor (HBV) diodes for frequency multiplication. The polarization charges do not deteriorate the performance of $\mathrm{GaN} / \mathrm{AlGaN} \mathrm{HBV}$ diodes.

*e-mail: reklaitis@pfi.lt 


\section{Model}

The Monte Carlo method used in the present work for the simulation of vertical transport in heterostructures is described in [1]. A three-nonparabolic valley conduction band model for GaN and AlGaN is used in the simulations. The valleys are represented by spherical dispersion relationships. The minimum of the conduction band is located at the center of the Brillouin zone ( $\Gamma_{1}$ point). In wurtzite $\mathrm{GaN}$, the higher valleys of the conduction band are located at $\Gamma$ point $\left(\Gamma_{2}\right)$ and $U$ point (located two thirds of the way between $L$ and $M$ symmetry points). The electron scattering mechanisms considered in the simulations include ionized impurity, polar optical, deformation acoustic, piezoelectric, and intervalley scattering. The numerical values of GaN material parameters are taken the same as in [2]. The investigated double-barrier structure consists of a repetition of a $15 \mathrm{~nm}$ undoped $\mathrm{Al}_{0.4} \mathrm{Ga}_{0.6} \mathrm{~N}$ barrier sandwiched between two $n$-GaN layers $(n=$ $1 \times 10^{17} \mathrm{~cm}^{-3}$ ) with a thickness of $250 \mathrm{~nm}$. Undoped $4 \mathrm{~nm}$ GaN spacer layers are inserted between the $n$-GaN layers and the barriers. The double-barrier structure is sandwiched between $n^{+}$-GaN contacts $\left(N_{\mathrm{d}}=5 \times 10^{18} \mathrm{~cm}^{-3}\right)$. The composition of the investigated structure is shown in the Table.

TABLE

Double-barrier $\mathrm{Al}_{0.4} \mathrm{Ga}_{0.6} \mathrm{~N} / \mathrm{GaN}$ structure.

\begin{tabular}{l|c|c}
\hline \hline \multicolumn{1}{c|}{ Layer } & Thickness $[\mathrm{nm}]$ & Doping concentration $\left[\mathrm{cm}^{-3}\right]$ \\
\hline$n^{+}$-GaN contact & & $5 \times 10^{18}$ \\
$\mathrm{GaN}$ & 250 & $1 \times 10^{17}$ \\
$\mathrm{GaN}$ spacer & 4 & undoped \\
$\mathrm{Al}_{0.4} \mathrm{Ga}_{0.6} \mathrm{~N}$ & 20 & undoped \\
$\mathrm{GaN}$ spacer & 4 & undoped \\
$\mathrm{GaN}$ & 250 & $1 \times 10^{17}$ \\
$\mathrm{GaN}_{\text {spacer }}$ & 4 & undoped \\
$\mathrm{Al}_{0.4} \mathrm{Ga}_{0.6} \mathrm{~N}$ & 20 & undoped \\
$\mathrm{GaN} \mathrm{spacer}_{\mathrm{GaN}}$ & 4 & undoped \\
$n^{+}$-GaN contact & 250 & $1 \times 10^{17}$
\end{tabular}

Heterojunctions are treated as abrupt interfaces. Electron thermionic emission over the barriers and tunneling through the barriers are taken into account. The triangular and trapezoidal shapes of potential barriers are considered for electron tunneling according to the energy of the impinging electron: trapezoidal for the low-energy values and triangular for the high-energy values, respectively. The transmission probabilities for tunneling through the triangular and trapezoidal barriers, which take into account the electron effective mass discontinuity at the 
heterointerfaces, are used in the model. The transmission probabilities are evaluated self-consistently with the instant potential profile.

The density of polarization charge at GaN/AlGaN heterointerfaces is taken from the experimental results presented in $[3,4]$. The experimentally determined offset between conduction bands of GaN and AlGaN is taken from [5].

\section{Current-voltage characteristic}

The calculated equilibrium potential and electron distribution in the double-barrier structure is presented in Fig. 1a. A very strong influence of polarization charges on the steady-state potential and electron distributions is evident from Fig. 1. The polarization charges induce the enhancement of barrier potential up to $4 \mathrm{eV}$ in equilibrium (Fig. 1a). As seen from comparison of Fig. $1 \mathrm{~b}$ and c, the polarization charges cause a strong asymmetry of potential distribution when diode is biased positively and negatively.
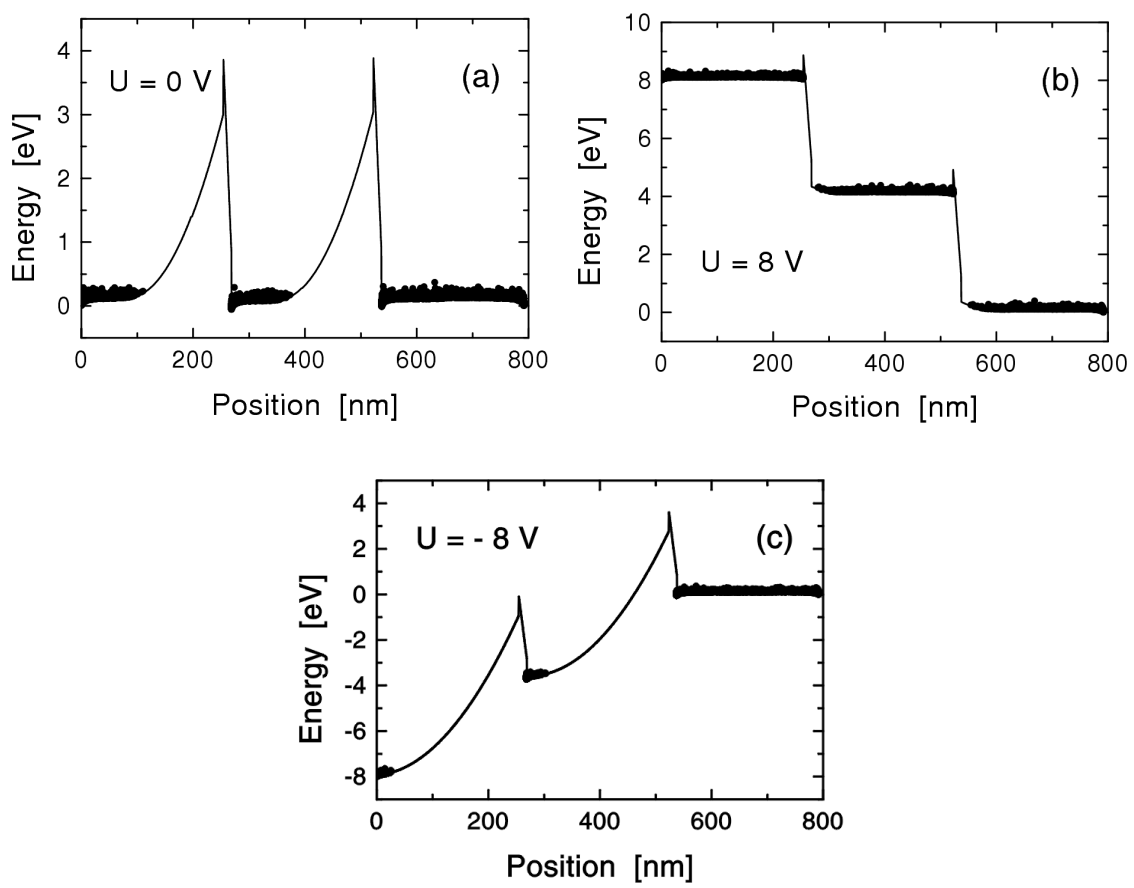

Fig. 1. Potential profile (solid curves) and distributions of electron kinetic energy (points) in double-barrier $\mathrm{GaN} / \mathrm{Al}_{0.4} \mathrm{Ga}_{0.6} \mathrm{~N}$ diode. (a) $U=0 \mathrm{~V}$ (equilibrium distribution). (b) $U=8 \mathrm{~V}$. (c) $U=-8 \mathrm{~V}$.

The calculated current-voltage $(I-V)$ characteristic of the single-barrier structure is presented in Fig. 2. The $I-V$ characteristic is strongly asymmetric due to polarization charges. The current in the positively biased diode essentially 


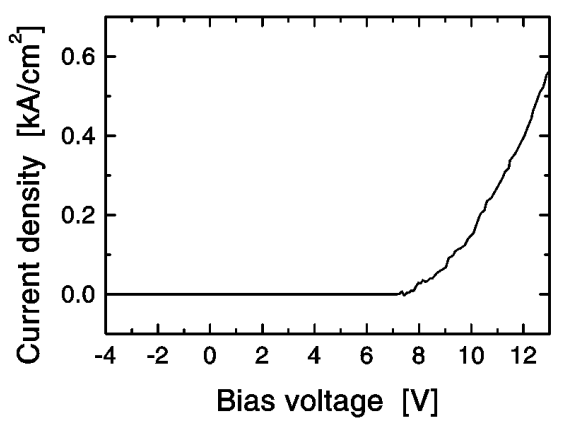

Fig. 2. Steady-state $I-V$ characteristic of double-barrier $\mathrm{GaN} / \mathrm{Al}_{0.4} \mathrm{Ga}_{0.6} \mathrm{~N}$ diode.

exceeds the current in negatively biased diode. The reason of asymmetry of $I-V$ characteristic can be explained by the potential distributions presented in Fig. 1. The results of simulations show that tunneling current controls the electron transport in the diode. The thermionic current over the barriers is negligible because the barrier height exceeds essentially the thermal energy of electrons. It can be seen from Fig. 1 that the barrier thickness of negatively biased diode exceeds essentially the barrier thickness of positively biased diode. This is the main reason for the strong asymmetry of $I-V$ characteristic.

\section{Capacitance--voltage characteristic}

The calculated capacitance-voltage $(C-V)$ characteristic of the double-barrier diode is shown in Fig. 3. Contrary to $I-V$ relationship, the $C-V$ characteristic is near symmetric with respect to non-zero bias voltage $U=7.5 \mathrm{~V}$. The comparison of $C-V$ characteristics calculated with and without polarization charges shows that polarization charges induce the shift of $C-V$ curve without substantial change in its shape. The $C-V$ characteristic presented in Fig. 3 demonstrates an excellent capacitance modulation ratio $C_{\max }: C_{\min }=8: 1$. As it is well known, the heterostructure diodes may operate as efficient frequency multipliers [6]. The

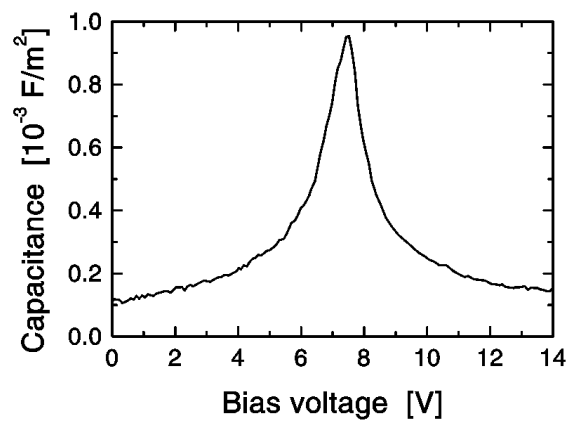

Fig. 3. $C-V$ characteristic of double-barrier $\mathrm{GaN} / \mathrm{Al}_{0.4} \mathrm{Ga}_{0.6} \mathrm{~N}$ diode. 
large capacitance modulation ratio implies that the $\mathrm{GaN} / \mathrm{Al}_{0.4} \mathrm{Ga}_{0.6} \mathrm{~N}$ structure is able to operate as efficient varactor diode in the frequency multiplication regime.

The results of simulations of the current response to sinusoidal excitation (Fig. 4) evidence that the current follows the steady-state $C-V$ relationship even though the excitation frequency is $100 \mathrm{GHz}$. This fact is confirmed by the calculated ratio of the amplitude of the third harmonic to the amplitude of the first harmonic. The harmonic amplitudes are calculated by Fourier transform of the current response to periodic excitation (Fig. 4). The ratio of the third harmonic amplitude to the first one is found to be 0.45 at $100 \mathrm{GHz}$ frequency of excitation signal. This result implies that the considered structure is able to operate as efficient frequency tripler in the submillimeter frequency range.

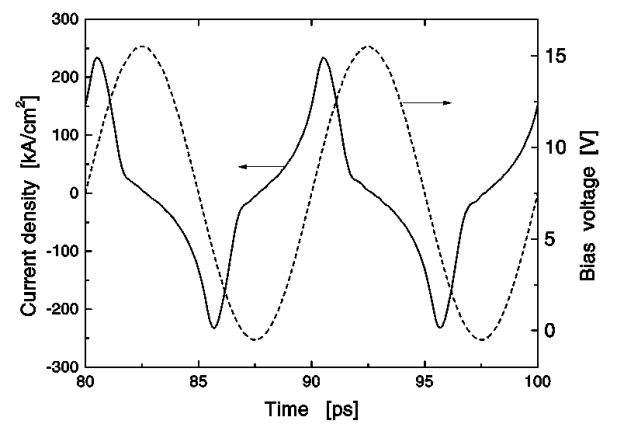

Fig. 4. Current response (solid curve) to the sinusoidal excitation of $100 \mathrm{GHz}$ frequency and $8 \mathrm{~V}$ amplitude (dashed curve). The dc bias voltage is $7.5 \mathrm{~V}$.

The results of experimental study of the double-barrier $\mathrm{Al}_{0.4} \mathrm{Ga}_{0.6} \mathrm{~N} / \mathrm{GaN}$ structure are reported in [7]. The composition of the structure experimentally investigated in [7] is the same as the one simulated in the present work except for the barrier thickness. The barrier thickness of the structure measured in [7] is increased to $20 \mathrm{~nm}$. The experimental results of [7] demonstrate the poor quality of the $C-V$ characteristic. The measured $C-V$ relationship has a small modulation ratio, and the $C-V$ characteristic is strongly asymmetric. Because of serious disagreement between the experimental results and the results of the simulations of the present work, we have simulated the double-barrier GaN/AlGaN structure measured in [3]. To this end, the barrier thickness is increased from $15 \mathrm{~nm}$ to $20 \mathrm{~nm}$. The calculated equilibrium potential and carrier distribution are presented in Fig. 5. The results of calculations show that in equilibrium, the Fermi energy at the heterointerfaces is below the top of the valence band. As a consequence the significant amount of holes exists in equilibrium. According to the calculations, the amount of holes exceeds $25 \%$ of the total amount of charge carriers in equilibrium. The holes may take a significant part in the formation of $I-V$ and $C-V$ characteristics of the structure because of shunting effect of the hole current. The simulations of the structures with different barrier thickness show that the 


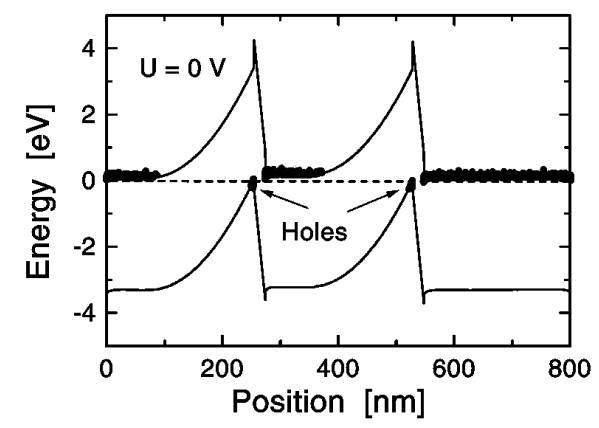

Fig. 5. Calculated equilibrium potential profile (solid curve) and distributions of carrier kinetic energy (points) in double-barrier diode with $20 \mathrm{~nm}$ barrier thickness. The Fermi energy is shown by dashed line. Arrows indicate the location of equilibrium holes.

thickness of the barriers of the structure measured in [3] should be reduced from $20 \mathrm{~nm}$ to at least $17 \mathrm{~nm}$ in order to prevent the excitation of equilibrium holes.

\section{Conclusions}

Due to polarization charges, the current-voltage $(I-V)$ characteristic of the symmetric multi-barrier GaN/AlGaN structures is strongly asymmetric. At certain conditions depending on $\mathrm{Al}$ mole fraction and barrier thickness, a considerable amount of holes is excited at heterointerfaces even in equilibrium.

Contrary to $I-V$ characteristic, the $C-V$ characteristic is near symmetric in respect to non-zero bias voltage. The calculated $C-V$ characteristic shows that GaN/AlGaN heterostructures are promising candidates for the design of efficient varactor diodes. The results of Monte Carlo studies of large-signal ac response of electrons in $\mathrm{GaN} / \mathrm{AlGaN}$ heterostructures demonstrate that GaN/AlGaN varactor diodes are capable to operate at higher frequency as compared to conventional InGaAs/AlAs and GaAs/AlGaAs varactor diodes.

\section{References}

[1] A. Reklaitis, G. Grigaliūnaite, Phys. Rev. B 63, 155301 (2001).

[2] A. Reklaitis, L. Reggiani, J. Appl. Phys. 95, 7925 (2004).

[3] V. Fiorentini, F. Bernardini, O. Ambacher, Appl. Phys. Lett. 80, 1204 (2002).

[4] E.J. Miller, E.T. Yu, C. Poblenz, C. Elsass, J.S. Speck, Appl. Phys. Lett. 80, 3551 (2002).

[5] D.R. Hang, C.H. Chen, Y.F. Chen, H.X. Jiang, J.Y. Lin, J. Appl. Phys. 90, 1887 (2001).

[6] E. Kolberg, A. Rydberg, Electron. Lett. 25, 1696 (1989).

[7] M. Saglam, K. Mutamba, A. Megej, C. Sydlo, H. Harnagel, Appl. Phys. Lett. 82, 227 (2003). 